\title{
Clinical Outcome of Percutaneous Trigeminal Nerve Block in Elderly Patients in Outpatient Clinics
}

\author{
Hyek Jun Seo, Chang Kyu Park, Man Kyu Choi, Jiwook Ryu, Bong Jin Park \\ Department of Neurosurgery, School of Medicine, Kyung Hee University, Seoul, Korea
}

Objective : Trigeminal neuralgia (TN) is a severe neuropathic condition that affects several elderly patients. It is characterized by uncontrolled pain that significantly impacts the quality of life of patients. Therefore, the condition should be treated as an emergency. In the majority of patients, pain can be controlled with medication; however, other treatment modalities are being explored in those who become refractory to drug treatment. The use of the trigeminal nerve block with a local anesthetic serves as an excellent adjunct to drug treatment. This technique rapidly relieves the patient of pain while medications are being titrated to effective levels. We report the efficacy and safety of percutaneous trigeminal nerve block in elderly patients with TN at our outpatient clinic.

Methods : Twenty-one patients older than 65 years with TN received percutaneous nerve block at our outpatient clinic. We used bupivacaine (1 $\mathrm{mL} /$ injection site) to block the supraorbital, infraorbital, superior alveolar, mental, and inferior alveolar nerves according to pain sites of patients.

Results : All patients reported relief from pain, which decreased by approximately $78 \%$ after 2 weeks of nerve block. The effect lasted for more than 4 weeks in 12 patients and for 6 weeks in two patients. There were no complications.

Conclusion : Percutaneous nerve block procedure performed at our outpatient clinic provided immediate relief from pain to elderly patients with TN. The procedure is simple, has no serious side effects, and is easy to apply.

Key Words : Trigeminal neuralgia · Nerve block · Bupivacaine · Emergency.

\section{INTRODUCTION}

Trigeminal neuralgia (TN) is a neuropathic condition characterized by recurrent brief episodes of sudden stabbing (lancinating) facial pain involving the trigeminal nerve. The condition is mostly unilateral and can involve one or more branches of the trigeminal nerve. The severe paroxysmal pain associated with the condition affects the physical functions of patients and reduces their quality of life ${ }^{15,25}$. TN predominantly occurs in individuals older than 60 years. Its onset is rare in individuals younger than 40 years, except in those with multiple sclerosis or cancer, where symptoms differ from the "classic" TN in terms of facial sensory loss and pain distribution along the branches of the trigeminal nerve ${ }^{4,22}$. The common age of onset of TN is

- Received : May 12, 2020 •Revised : July 15, 2020 •Accepted : August 17, 2020

- Address for reprints : Chang Kyu Park

Department of Neurosurgery, School of Medicine, Kyung Hee University, 23 Kyungheedae-ro, Dongdaemun-gu, Seoul 02447, Korea

Tel : +82-2-958-8385, Fax : +82-2-958-8380, E-mail : changchaz@naver.com, ORCID : https://orcid.org/0000-0002-5935-8264

This is an Open Access article distributed under the terms of the Creative Commons Attribution Non-Commercial License (http://creativecommons.org/licenses/by-nc/4.0) which permits unrestricted non-commercial use, distribution, and reproduction in any medium, provided the original work is properly cited. 
40 to 60 years; however, years or even decades pass before the initially brief episodes of pain become intractably severe or frequent and unresponsive to medication. A majority of patients seek first neurosurgical intervention for their symptoms in their eighth decade of life. Moreover, several patients at this age or above continue to seek neurosurgical assistance when their $\mathrm{TN}$ fails to respond to initial surgery or recurs after transient success ${ }^{19)}$.

Immediate surgical intervention is not possible in elderly patients presenting with severe facial pain. There is a growing concern of complications associated with surgery with the increase in age. Furthermore, elderly patients find it difficult to wait for surgical treatment ${ }^{19)}$. Therefore, other treatment approaches, such as percutaneous administration of medicines, are being used, which are relatively easy to apply and more effective. However, these require expert technical skills. Furthermore, several of these are neurodestructive in nature and may be associated with adverse side effects such as sensory loss and dysesthesia ${ }^{7)}$.

This study investigated the clinical outcome of percutaneous nerve block of the trigeminal nerve in terms of its efficacy and safety in elderly patients with TN in our outpatient clinic.

\section{MATERIALS AND METHODS}

All procedures performed in studies involving human participants were in accordance with the ethical standards of the Institutional Research Committee of Kyung Hee University Hospital (approval number KMC IRB 1511-14) and with the 1964 Helsinki declaration and its later amendments or comparable ethical standards.

\section{Patient and study design}

This study was conducted from January 2018 to December 2019 on patients older than 65 years who were treated for TN in our hospital. These patients did not show an improvement in symptoms with medication and thus received additional injection therapy in the outpatient facility.

Patients included in the study visited the outpatient clinic every 2 weeks after injection therapy for assessment of the degree of pain. Numeric rating scale (NRS) was used to measure the degree of pain. The patients were maintained on medication even after the injection therapy. No additional injection treatments were administered during the 6 weeks of outcome evaluation.

\section{Intervention}

All nerve block procedures were performed with bupivacaine hydrochloride $(0.5 \%, 5 \mathrm{mg} / \mathrm{mL})$ using a 23 -gauge needle. In the majority of patients, $1 \mathrm{~mL}$ of the drug was administered at each injection site.

The majority of the ophthalmic (V1) division of the trigeminal nerve had a supraorbital nerve block. In the case of maxillary (V2) division, the infraorbital and superior alveolar nerves were blocked, whereas in the case of mandibular (V3) division, the mental and inferior alveolar nerves were blocked. Neuroblocking was performed according to the previously described procedure ${ }^{6,10,12)}$.

The supraorbital nerve is one of the terminal branches of the trigeminal-ophthalmic nerve. It exits the cranium via an opening above the orbit known as the supraorbital foramen. It is visually identified by asking the patient to look straight ahead and transect the pupils at the level of the orbital ridge. Palpation of this region identifies the injection site for performing this nerve block.

The infraorbital nerve is a branch of the maxillary nerve, which is the second division of the trigeminal nerve. The key injection site for performing an infraorbital nerve block is the infraorbital foramen. The injection site is identified by asking the patient to look straight ahead and imagine a line down from the pupils to the inferior border of the infraorbital ridge, bicuspid teeth, and mental foramen.

The mental nerve is one of the branches of the inferior alveolar nerve. It exits through the mental foramen bilaterally in the mandible. The mental foramen is located halfway between the upper (alveolar crest) and the lower edges of the mandible in direct line with the second bicuspid (premolar). Two techniques are used to perform this nerve block : intraoral and extraoral (percutaneous). We selected the intraoral procedure. The mental foramen was identified by asking the patient to retract the lower lip.

The superior alveolar nerve is divided into three branches, namely anterior, superior, and alveolar nerves. It descends from the infraorbital nerve and innervates the ipsilateral incisors and the canine. The nerve block is performed using the intraoral method. After the upper lip is retracted anteriorly and superiorly, the injection is administered at an angle of 45 
degrees on the apex of the canine. The middle superior alveolar nerve is a branch of the infraorbital nerve or the maxillary nerve. This nerve innervates the ipsilateral premolars and the first molar. The injection site is between the apices of premolar and the first molar, and injection is administered at an angle of 45 degrees. The posterior superior alveolar nerve is a branch of the maxillary nerve and innervates the ipsilateral molars. The injection site is the root of the upper secondary molar.

The inferior alveolar nerve is a branch of the mandibular nerve. It is blocked using the intraoral method. After the pterygomandibular triangle is checked, the syringe is held parallel to the occlusal surfaces of the teeth and at an angle such that the barrel lies between the 1st/2nd premolars of the opposite side. The injection is administered between the pterygomandibular raphe and the coronoid notch.

Patients were returned home after confirming that a response appeared within 15 minutes after injection treatment.

\section{RESULTS}

A total of 21 patients were included in the study. The mean age was $71.8 \pm 4.7$ years (range, $65-82$ ). There were nine men and 13 women. The median duration of symptoms was 3 months, ranging from 2 weeks to 9 months. All patients received no treatment other than medication (Table 1).

All patients underwent outpatient follow-up for more than 6 weeks. Pain distribution was V1 in one, V1+2 in two, V2 in $11, \mathrm{~V} 2+3$ in five, and V3 in two patients. All patients who received the injection felt a decrease in sensation in the pain area within 15 minutes after the injection.

The mean NRS of patients at the time of the visit was 7.2 \pm 0.8 points (range, $6-9$ ). The mean score measured 2 weeks after the procedure was $1.6 \pm 1.1$ (range, $0-4$ ), showing that the pain reduced by approximately $78 \%$. The mean score measured after 1 month of the procedure was 3.14 \pm 2.1 (range,

Table 1. Demographics of patients

\begin{tabular}{|c|c|c|c|c|c|}
\hline Case No. & Age & Sex & Distribution of pain & Medication (daily dose) & $\begin{array}{l}\text { Interval time between injection } \\
\text { and symptom onset }\end{array}$ \\
\hline 1 & 68 & M & V2 & Carbamazepine 400 mg/gabapentin 200 mg & 1 month \\
\hline 2 & 70 & $\mathrm{~F}$ & V2 & Carbamazepine 400 mg & 3 months \\
\hline 3 & 65 & $\mathrm{~F}$ & $\mathrm{~V} 2+\mathrm{V} 3$ & Carbamazepine 400 mg/gabapentin 600 mg & 1 month \\
\hline 4 & 71 & M & $\mathrm{V} 2+\mathrm{V} 3$ & Carbamazepine 400 mg/gabapentin 600 mg & 5 months \\
\hline 5 & 70 & $\mathrm{~F}$ & V1 & Carbamazepine 400 mg/gabapentin 200 mg & 4 months \\
\hline 6 & 78 & M & V2 & Carbamazepine 600 mg/gabapentin 600 mg & 3 months \\
\hline 7 & 65 & M & V2 & Carbamazepine 400 mg/gabapentin 200 mg & 2 weeks \\
\hline 8 & 70 & $\mathrm{~F}$ & V2 & Carbamazepine 200 mg/gabapentin 200 mg & 9 months \\
\hline 9 & 69 & $\mathrm{~F}$ & V2 & Carbamazepine 400 mg/gabapentin 200 mg & 6 months \\
\hline 10 & 70 & M & V2 & Carbamazepine 400 mg/gabapentin 600 mg & 3 months \\
\hline 11 & 75 & M & $\mathrm{V} 2+\mathrm{V} 3$ & Carbamazepine 400 mg/gabapentin 600 mg & 4 months \\
\hline 12 & 72 & $\mathrm{~F}$ & $\mathrm{~V} 2+\mathrm{V} 3$ & Gabapentin $600 \mathrm{mg}$ & 4 months \\
\hline 13 & 75 & $\mathrm{~F}$ & $\mathrm{~V} 2+\mathrm{V} 3$ & Carbamazepine 600 mg/gabapentin 600 mg & 3 months \\
\hline 14 & 72 & M & V3 & Carbamazepine 400 mg/gabapentin 200 mg & 1 month \\
\hline 15 & 65 & $\mathrm{~F}$ & V2 & Carbamazepine 400 mg/gabapentin 600 mg & 4 months \\
\hline 16 & 68 & F & V2 & Carbamazepine 200 mg & 1 month \\
\hline 17 & 80 & M & V2 & Carbamazepine 200 mg/gabapentin 200 mg & 9 months \\
\hline 18 & 82 & M & $\mathrm{V} 1+\mathrm{V} 2$ & Carbamazepine 400 mg/gabapentin 200 mg & 2 months \\
\hline 19 & 75 & $\mathrm{~F}$ & V2 & Carbamazepine 400 mg/gabapentin 200 mg & 1 month \\
\hline 20 & 75 & $\mathrm{~F}$ & V3 & Carbamazepine 400 mg/gabapentin 600 mg & 2 months \\
\hline 21 & 74 & $\mathrm{~F}$ & $\mathrm{~V} 1+\mathrm{V} 2$ & Carbamazepine 600 mg/gabapentin 200mg & 4 months \\
\hline
\end{tabular}

$\mathrm{M}$ : male, V2 : maxillary zone, F : female, V3 : mandibular zone, V1 : ophthalmic zone 
$1-7)$, and that evaluated 6 weeks after the procedure was $5.7 \pm$ 1.6 (range, 2-8). The change in the pain score after injection therapy showed statistical significance $(p<0.0 .001)$ (Fig. 1).

Although patients were divided according to the nerve branch blocked, the majority of them reported effective pain reduction after the procedure (Fig. 2). The average NRS of four patients who received supraorbital nerve block was 8 points before the injection, which reduced to 1 point 2 weeks later. After 4 weeks, the mean NRS was 2 points and increased to 5 points after 6 weeks. The average NRS of seven patients who received infraorbital nerve block was 7 points before the injection; it reduced to 2 points 2 weeks later. After 4 weeks, the mean NRS was 4 points and increased to 5.5 points after 6 weeks. The initial NRS score of five patients who received superior alveolar nerve block was 7 points, which reduced to 1.9 points after 2 weeks. The mean NRS was 2.2 points after 4 weeks and 5 points after 6 weeks. The initial NRS of four patients who received inferior alveolar nerve block was 7 points. It decreased to 1 point after 2 weeks, and the NRS was 3 points after 4 weeks and increased to 8 points after 6 weeks. The initial NRS score of four patients who received the mental nerve block was 9 points. The score decreased dramatically to 0.5 points after 2 weeks; however, the NRS increased dramatically to 7 points after 4 weeks. There was no change after 6 weeks. In the majority of patients, the best effect was observed 2 weeks after the procedure, and the pain score increased after 2 weeks. The duration of pain improvement lasted for more than 4 weeks in 12 patients and 6 weeks in two patients. No complications were reported.

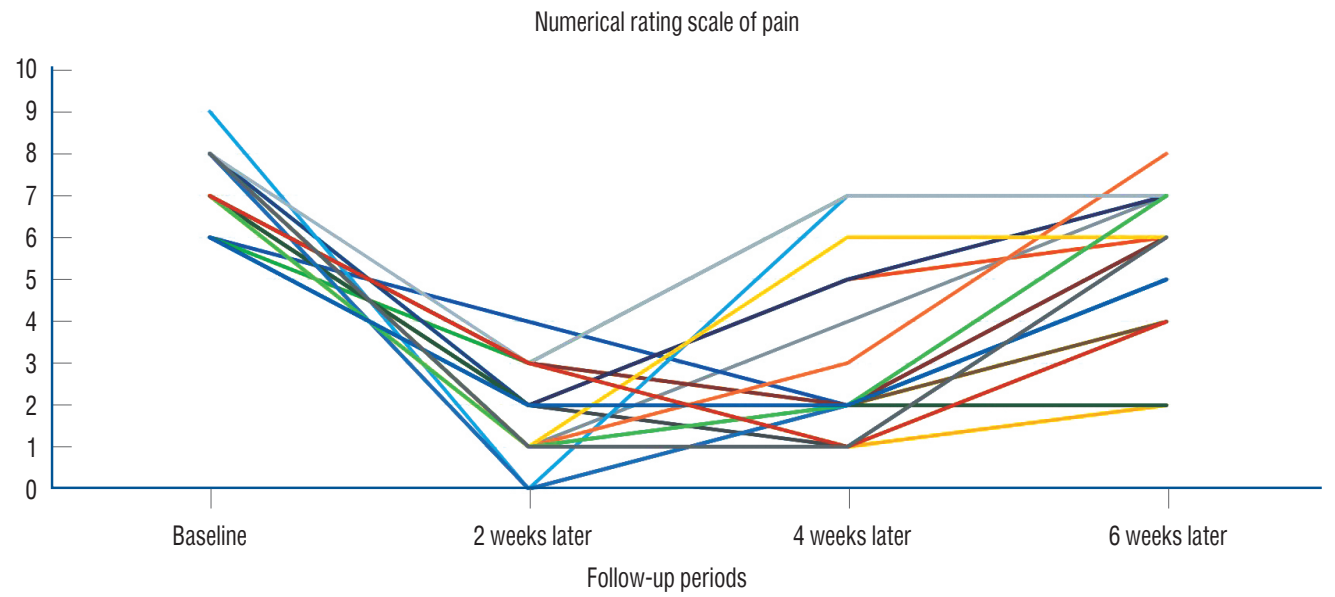

Fig. 1. Score after treatment of each patient.

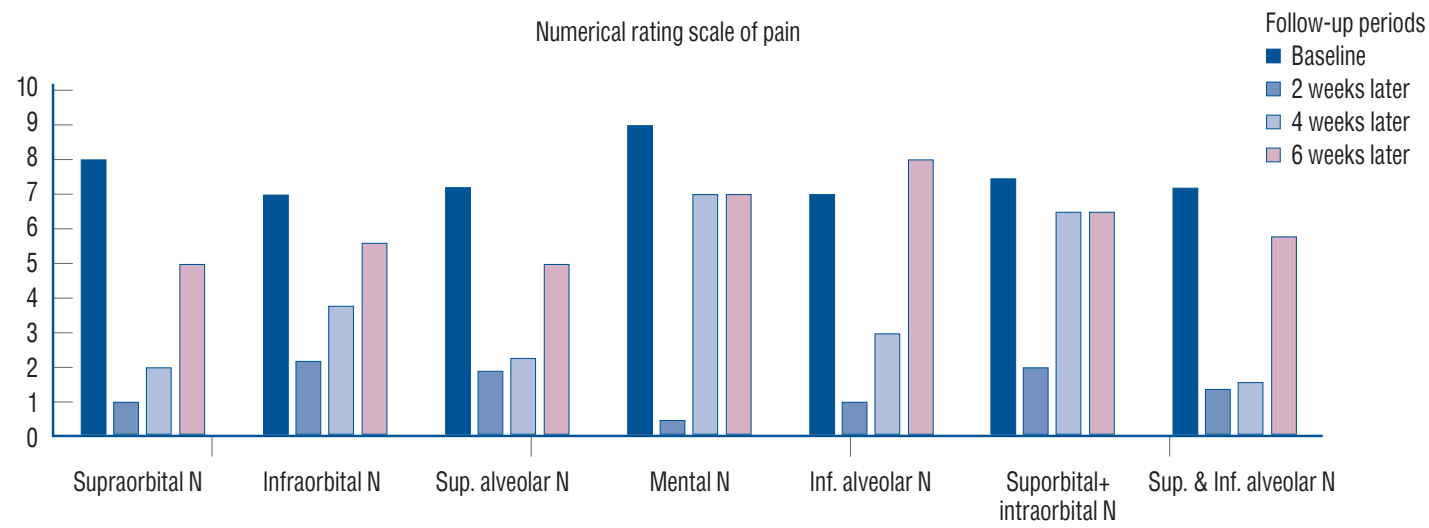

Fig. 2. Score after treatment of each branch of trigeminal nerve. $\mathrm{N}$ : nerve, Sup. : superior, Inf. : inferior. 


\section{DISCUSSION}

Medication is a commonly used treatment for TN. However, in patients refractory to drug treatment, other treatment modalities are explored. Surgery is the commonly used treatment if the cause of the disease is a neurovascular conflict. Microvascular decompression is considered the best surgical treatment for TN owing to its effectiveness and durability ${ }^{1,16)}$. Other treatment approaches include radiofrequency ablation of the trigeminal ganglion and gamma knife radiosurgery $(\mathrm{GKS})^{2,17)}$.

A systematic review reported a mean postoperative success rate of $83.5 \%$ for microvascular decompression ${ }^{24)}$. Moreover, the incidence of complications related to the surgery was approximately $1 \%$ to $2 \%{ }^{211}$. According to a study, acute pain relief was experienced by $97.6 \%$ of patients after radiofrequency ablation $^{14}$. Another study reported that $57.7 \%$ of patients experienced complete pain relief 5 years after ablation ${ }^{9}$. Marshall et al. ${ }^{18)}$ reported that the treatment response rate was $86 \%$ within 3 months of GKS. Of these, $43 \%$ of patients experienced excellent outcomes ${ }^{18)}$.

In elderly patients, microvascular decompression surgery can be performed in those fit for general anesthesia. In addition, in the case of complications following the surgery, the sequelae are serious such that the decision of surgery should be made carefully $y^{3}$. Radiofrequency ablation requires the operator's proficiency. Moreover, it is associated with side effects such as facial sensory disorders, e.g., hypoesthesia ${ }^{11}$. In addition, the effect of treatment is not permanent leading to additional or re-treatments. In the case of GKS, a latent period is required for the treatment to exert its effect, which does not last long ${ }^{17)}$.

Peripheral blockage of the trigeminal nerve branches has the advantage that it exerts an immediate effect that lasts for at least 2 weeks. Furthermore, it can be performed immediately in an outpatient setting due to the ease of the procedure. It has few side effects and can be easily applied to elderly patients. In addition, the bupivacaine monotherapy performed in this study has fewer side effects than the conventional cocktail therapy, with the advantage of re-injection within a short duration depending on the treatment response.

The major disadvantage of injection therapy is that its duration of action is short. In addition, it is difficult to objectively estimate the duration of the treatment effect on different patients.
However, injection therapy immediately exerts the desired therapeutic effect on patients with severe pain in an outpatient setting, enabling continuous administration of medication.

NRS of the patients were reduced by $78 \%$ after 2 weeks of treatment in the present study. It is commonly reported that bupivacaine has a working time of $2-8$ hours $^{13)}$. In the current study, the duration of the action for about two weeks is not just the injection effect. We thought that it is because the additional medication was treated together. Bupivacaine has the following mechanism of action. It binds to the intracellular portion of voltage-gated sodium channels and blocks sodium influx into nerve cells, which prevents depolarization. Without depolarization, no initiation or conduction of a pain signal can occur. Moreover, carbamazepine, which is most commonly used for TN, is also a sodium channel blocker, so it can be cautiously speculated that the mechanism of drug action was synergistic with each other ${ }^{5}$. In addition, the duration of action may be seen as extended by the implementation of additional drug administration and dose increases because they were not controlled by conventional drugs.

Drugs in injection treatment may use other drugs than bupivacaine. Wilkinson ${ }^{23)}$ performed trigeminal peripheral nerve block using phenol/glycerol. As a result, they reported pain relief in about $87 \%$ of patients within 24 hours. They also reported that $37 \%$ of patients still relieved after a year. However, side effects such as facial palsy, ecchymosis and facial sensory disorders were also reported. Fernandez et al. ${ }^{8)}$ reported that the action time of bupivacaine was about twice as long as the result of comparative analysis of bupivacaine and lidocaine. Perloff and Chung ${ }^{20)}$ were injected with a mixture of 2 $\mathrm{mL}$ bupivacaine and $1 \mathrm{~mL}$ lidocaine in patients with $\mathrm{TN}$ to evaluate the response. They reported that all of nine patients had a pain relief of more than $50 \%$ immediately after the procedure and the effect lasted more than three months in five of the patients.

This study has certain limitations. First, it was difficult to objectively verify the duration of treatment because it was conducted with a small number of patients. Second, there was heterogeneity among the study subjects as the analysis was not performed according to the drugs administered to patients. Further studies using large patient sample size are required to objectively analyze the treatment duration and effectiveness of injection therapy. 


\section{CONCLUSION}

$\mathrm{TN}$ is a neuropathic condition that is associated with severe pain. Medical treatment is effective; however, patients sometimes become refractory to drug treatments. In such cases, drugs could be administered as percutaneous injections to immediately subside pain in an outpatient clinic. The procedure is simple, has few side effects, and can be easily applied to elderly patients in whom the condition is difficult to treat using other treatment methods.

\section{CONFLICTS OF INTEREST}

No potential conflict of interest relevant to this article was reported.

\section{INFORMED CONSENT}

Informed consent was obtained from all individual participants included in this study.

\section{AUTHOR CONTRIBUTIONS}

\author{
Conceptualization : CKP \\ Data curation : CKP, BJP \\ Formal analysis : HJS, CKP \\ Methodology : MKC, JR \\ Project administration : CKP, BJP \\ Visualization : HJS, CKP \\ Writing - original draft : HJS \\ Writing - review \& editing : CKP
}

\section{ORCID}

$\begin{array}{ll}\text { Hyek Jun Seo } & \text { https://orcid.org/0000-0002-9236-8720 } \\ \text { Chang Kyu Park } & \text { https://orcid.org/0000-0002-5935-8264 } \\ \text { Man Kyu Choi } & \text { https://orcid.org/0000-0001-6670-7541 } \\ \text { Jiwook Ryu } & \text { https://orcid.org/0000-0001-5091-6760 } \\ \text { Bong Jin Park } & \text { https://orcid.org/0000-0002-6486-1681 }\end{array}$

\section{References}

1. Amirnovin R, Neimat JS, Roberts JA, Eskandar EN : Multimodality treatment of trigeminal neuralgia. Stereotact Funct Neurosurg 83 : 197201, 2005

2. Arıcı T, Kurcaloğlu M, Kılıç E, Erhan E : Radiofrequency thermocoagulation combined with pulsed radiofrequency for gasserian ganglion blockage. Agri 30 : 179-182, 2018

3. Ashkan $\mathrm{K}$, Marsh $\mathrm{H}$ : Microvascular decompression for trigeminal neuralgia in the elderly: a review of the safety and efficacy. Neurosurgery 55 : 840-848; discussion 848-850, 2004

4. Bahgat D, Ray DK, Raslan AM, McCartney S, Burchiel KJ : Trigeminal neuralgia in young adults. J Neurosurg 114 : 1306-1311, 2011

5. Bertilsson L : Clinical pharmacokinetics of carbamazepine. Clin Pharmacokinet 3 : 128-143, 1978

6. Dach F, Éckeli ÁL, Ferreira Kdos S, Speciali JG : Nerve block for the treatment of headaches and cranial neuralgias - a practical approach. Headache 55 Suppl $1:$ 59-71, 2015

7. Emril DR, Ho KY : Treatment of trigeminal neuralgia: role of radiofrequency ablation. J Pain Res 3 : 249-254, 2010

8. Fernandez C, Reader A, Beck M, Nusstein J : A prospective, randomized, double-blind comparison of bupivacaine and lidocaine for inferior alveolar nerve blocks. J Endod 31 : 499-503, 2005

9. Fraioli MF, Cristino B, Moschettoni L, Cacciotti G, Fraioli C : Validity of percutaneous controlled radiofrequency thermocoagulation in the treatment of isolated third division trigeminal neuralgia. Surg Neurol 71 : 180-183, 2009

10. Hascalovici JR, Robbins MS : Peripheral nerve blocks for the treatment of headache in older adults: a retrospective study. Headache $57: 80$ 86,2017

11. Hong T, Ding Y, Yao P : Long-term efficacy and complications of radiofrequency thermocoagulation at different temperatures for the treatment of trigeminal neuralgia. Biochem Res Int 2020 : 3854284, 2020

12. Iffeld BM : Continuous peripheral nerve blocks: a review of the published evidence. Anesth Analg 113 : 904-925, 2011

13. Ilfeld BM, Viscusi ER, Hadzic A, Minkowitz HS, Morren MD, Lookabaugh J, et al. : Safety and side effect profile of liposome bupivacaine (exparel) in peripheral nerve blocks. Reg Anesth Pain Med 40 : 572-582, 2015

14. Kanpolat $Y$, Savas A, Bekar A, Berk C : Percutaneous controlled radiofrequency trigeminal rhizotomy for the treatment of idiopathic trigeminal neuralgia: 25-year experience with 1,600 patients. Neurosurgery 48 : 524-532; discussion 532-534, 2001

15. Maarbjerg S, Di Stefano G, Bendtsen L, Cruccu G : Trigeminal neuralgia diagnosis and treatment. Cephalalgia 37 : 648-657, 2017

16. Maarbjerg S, Heinskou TB, Wolfram F, Rochat P, Brennum J, Bendtsen L : Diagnostics and treatment of trigeminal neuralgia. Ugeskr Laeger 178 : V02160146, 2016

17. Marchetti M, Pinzi V, De Martin E, Ghielmetti F, Fariselli L : Radiosurgery for trigeminal neuralgia: the state of art. Neurol Sci 40(Suppl 1) : 153-157, 2019

18. Marshall K, Chan MD, McCoy TP, Aubuchon AC, Bourland JD, McMullen 
$\mathrm{KP}$, et al. : Predictive variables for the successful treatment of trigeminal neuralgia with gamma knife radiosurgery. Neurosurgery 70 : 566572; discussion 572-573, 2012

19. Oomens MA, Forouzanfar $T$ : Pharmaceutical management of trigeminal neuralgia in the elderly. Drugs Aging 32 : 717-726, 2015

20. Perloff MD, Chung JS : Urgent care peripheral nerve blocks for refractory trigeminal neuralgia. Am J Emerg Med 36 : 2058-2060, 2018

21. Sarsam Z, Garcia-Fiñana M, Nurmikko TJ, Varma TR, Eldridge $P$ : The long-term outcome of microvascular decompression for trigeminal neuralgia. Br J Neurosurg 24 : 18-25, 2010

22. Stienen MN, Cadosch D, Seule MA, Fournier JY, Hildebrandt G, Gautschi
OP : Trigeminal neuralgia - pathophysiology, clinical aspects and treatment. Praxis (Bern 1994) 99 : 29-43, 2010

23. Wilkinson HA : Trigeminal nerve peripheral branch phenol/glycerol injections for tic douloureux. J Neurosurg 90 : 828-832, 1999

24. Xia L, Zhong J, Zhu J, Wang YN, Dou NN, Liu MX, et al. : Effectiveness and safety of microvascular decompression surgery for treatment of trigeminal neuralgia: a systematic review. J Craniofac Surg 25 : 14131417, 2014

25. Yadav YR, Nishtha Y, Sonjjay P, Vijay P, Shailendra R, Yatin K : Trigeminal neuralgia. Asian J Neurosurg 12 : 585-597, 2017 